\title{
Case Report: Atypical Fibroxanthoma and Pleomorphic Dermal Sarcoma - Two Stages of the Same Disease?
}

\author{
Farhana Kapasi ${ }^{1 *}$, Marta Cabral' ${ }^{2}$, Phillip Ameerally ${ }^{1}$ and Antonia Barbieri'
}

${ }^{1}$ Northampton General Hospital, UK

${ }^{2}$ The Royal Devon and Exeter NHS Foundation Trust, UK

\section{Introduction}

Atypical Fibroxanthoma is a rare cutaneous tumour first described in 1963 by Elson Helwig [1]. It has been related to ultraviolet-induced damage $[2,3]$ and most frequently occurs in sun damaged head and neck skin [4-6]. Other risk factors include trauma, burns [7], irradiation, xeroderma pigmentosum, and organ transplantation [8].

The affected individuals often have a previous history of squamous cell carcinoma, basal cell carcinoma or actinic keratosis [9]. It affects mostly males and tends to occur more frequently in the seventh decade [2]. In 2010, a study of 171 cases of Atypical Fibroxanthoma reported that $91 \%$ of the tumours occurred on the head and neck, with the remaining $9 \%$ presenting on the extremities and trunk and $99 \%$ occurred on sun-damaged skin. The majority of tumours occurred in men $(76 \%)$, with a mean age of 74 years [10].

Clinically, Atypical Fibroxanthoma presents as a red, pink or purple nodule usually less than $2 \mathrm{~cm}$ in diameter. It is rapidly growing and can also present with ulceration and bleeding. Its clinical appearance may be confused with squamous cell carcinoma or basal cell carcinoma $[3,6]$.

It is considered a tumour with malignant histopathological features but a benign clinical course $[6,10,11]$. The treatment of choice is complete excisional biopsy with adequate margins $[2,3,11]$. Acceptable surgical margins vary from $3 \mathrm{~mm} \mathrm{[6]} \mathrm{to} 20 \mathrm{~mm} \mathrm{[12].}$

Recurrence rates of 5\% [13] to 10\% [14,15] following wide excision have been reported. Recurrence rates with incomplete margins can be as high as $20 \%$ [2]. Local recurrence appears to have a positive correlation with the development of distant metastatic disease [16]. Metastasis are rare, and the exact rate is unknown, although it is estimated to occur in $1 \%$ of cases [2] and it seems to occur usually within 20 months after the initial diagnosis [16].

Atypical Fibroxanthoma and Pleomorphic Dermal Sarcoma have similar histological features and can be difficult to differentiate. Advancements in immunohistochemistry have emphasised the similarities [3] with some studies advocating that they may represent two stages of the same disease rather than two different entities [17]. A study from 2016 has raised the hypotheses that Atypical Fibroxanthoma is the non-infiltrating precursor lesion of Pleomorphic Dermal Sarcoma [18]. Atypical Fibroxanthoma is confined to the dermis and does not share some of the adverse histopathological features of Pleomorphic Dermal Sarcoma, such as deep subcutaneous invasion, tumor necrosis and perineural and/ or lymphovascular invasion [9].

Differentiating between Atypical Fibroxanthoma and Pleomorphic Dermal Sarcoma is pivotal as studies have shown that Pleomorphic Dermal Sarcoma represents an inherently higher risk. For these tumours, recent literature has suggested that imaging and SLNB may be helpful in select cases. Radiation therapy may also be of adjuvant benefit for these tumors when clear surgical margins cannot be obtained [19].

The recent discovery of key oncogenetic mutations

*Corresponding author: Farhana Kapasi, MBBS, BDS, BSc (Hons), MRCS Northampton General Hospital, Cliftonville NN1 5BD Northampton, United Kingdom, Tel: +447838840937

Accepted: September 17, 2018;

Published online: September 19, 2018

Citation: Kapasi F, Cabral M, Ameerally P, et al. (2018) Case Report: Atypical Fibroxanthoma and Pleomorphic Dermal Sarcoma - Two Stages of the Same Disease?. Arch Surg Dermatol 1(1):13-17

Copyright: (c) 2018 Kapasi F, et al. This is an open-access article distributed under the terms of the Creative Commons Attribution License, which permits unrestricted use, distribution, and reproduction in any medium, provided the original author and source are credited. 
Citation: Kapasi F, Cabral M, Ameerally P, et al. (2018) Case Report: Atypical Fibroxanthoma and Pleomorphic Dermal Sarcoma - Two Stages of the Same Disease?. Arch Surg Dermatol 1(1):13-17

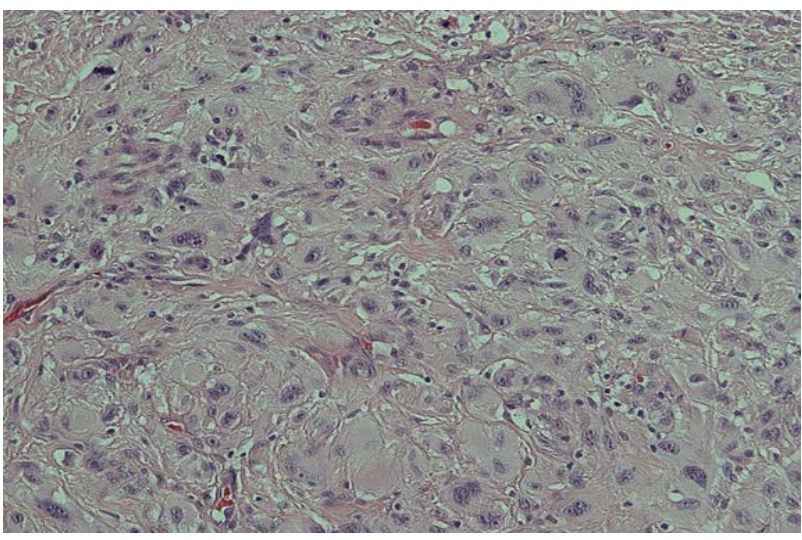

Figure 1: Histological image of the tumour, showing mainly atypical pleomorphic polygonal and some spindleshaped cells (Light microscopy $\mathrm{x} 20$; haematoxylin and eosin stain).

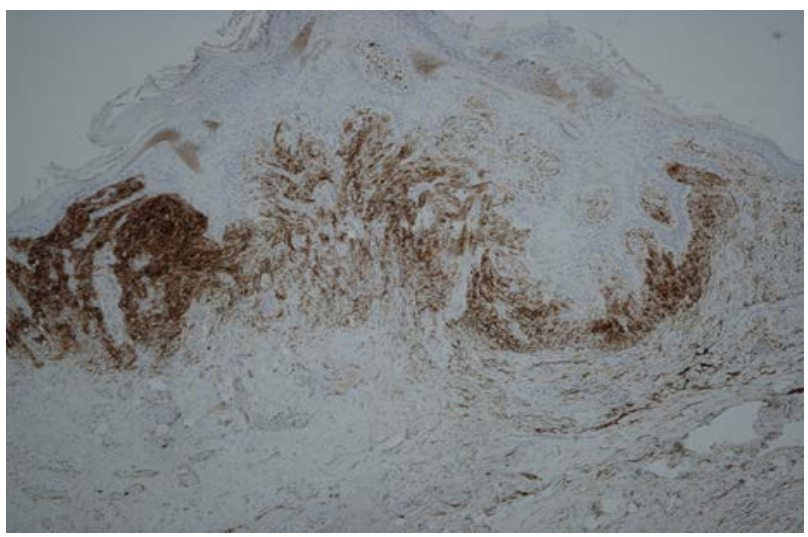

Figure 2: Immunohistochemical stain - tumour cells stain with CD10 (Light microscopy x4).

has allowed for the identification of several potential molecular drug targets that may have a therapeutic role with future study, however further studies are needed to establish definitive conclusions regarding risk stratification and best management practices [19].

\section{Case Report}

In November 2015, an 83-year-old male with a history of cataracts and multiple sites of actinic keratosis in the head treated with cryotherapy, presented with a $7 \mathrm{~mm}$ by $5 \mathrm{~mm}$ erythematous papule on the right parietal scalp, located closely to a pigmented macule which had been previously biopsied with a diagnosis of lentigo simplex. There had been previous attempts to treat this lesion with cryotherapy which were unsuccessful. An excisional biopsy was planned and performed in December 2015 , with secondary intention healing.

The histopathological report showed an expansile dermal nodule arising in sun-damaged skin comprising highly atypical and pleomorphic round and focally spindle-shaped cells showing mitosis (Figure 1). There was no

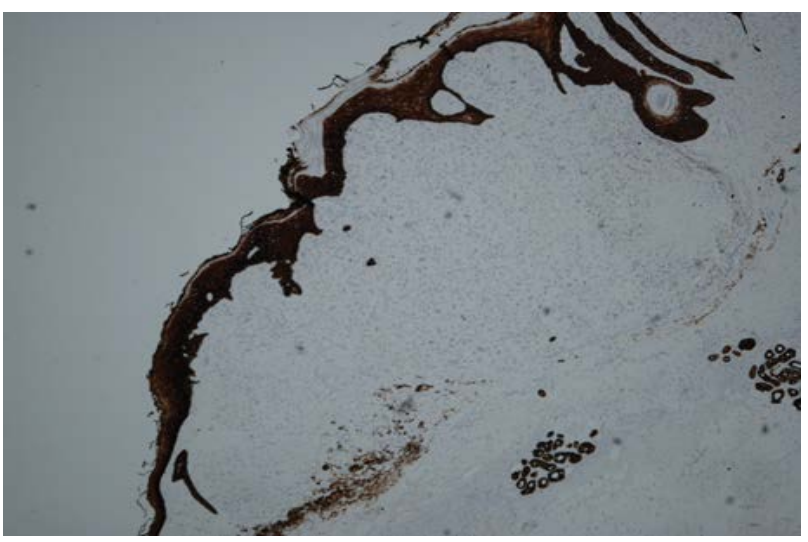

Figure 3: Immunohistochemical stain - tumour cells are negative with pancytokeratin MNF116 (Light microscopy x4).

evidence of necrosis. The nodule was limited to the dermis and there was no extension into the deep dermis or subcutaneous tissue. There was no evidence of lymphovascular or perineural invasion. In the background skin there was marked sun damage in the form of atrophic epidermis, solar elastosis and also foci of actinic keratosis. Immunohistochemistry was performed. The lesional cells were positive for CD10 (Figure 2), CD99 and CD68 (focal) and the spindle cell areas were positive for SMA (actin). The lesional cells were negative for cytokeratin 5, 34betaE12, MNF116 (Figure 3), Pancytokeratin AE1/3, Melan-A, S-100, HMB45, Desmin and H-Caldesmon. The morphological features, taken together with the above immunohistochemical phenotype are those of Atypical Fibroxanthoma of the skin. The excision was complete with clear peripheral and deep margins.

At follow up in February 2016 there was pus and granulation tissue at the operative site. There was suspicion of associated erosive pustular dermatosis, which was managed with a topical steroid cream.

At further review in May 2016 there were signs of wound healing and in early July 2016, the wound had healed completely.

However, in late July 2016, the patient presented with a rapidly growing purple nodule measuring $3.5 \mathrm{~cm}$ in diameter which had developed in the healed wound, raising suspicions of a local recurrence of the Atypical Fibroxanthoma or presence of a Squamous Cell Carcinoma. No regional lymphadenopathy was present.

The patient underwent excisional biopsy (Figure 4). The lesion was adherent to the underlying skull with diploic invasion and there was a full thickness bony defect (Figure 5). The defect was covered with a full thickness skin graft taken from the left neck as a temporising measure.

A subsequent CT scan showed a large bony defect in the right posterior parietal region consistent with the tumour invasion (Figure 6). 
Citation: Kapasi F, Cabral M, Ameerally P, et al. (2018) Case Report: Atypical Fibroxanthoma and Pleomorphic Dermal Sarcoma - Two Stages of the Same Disease?. Arch Surg Dermatol 1(1):13-17

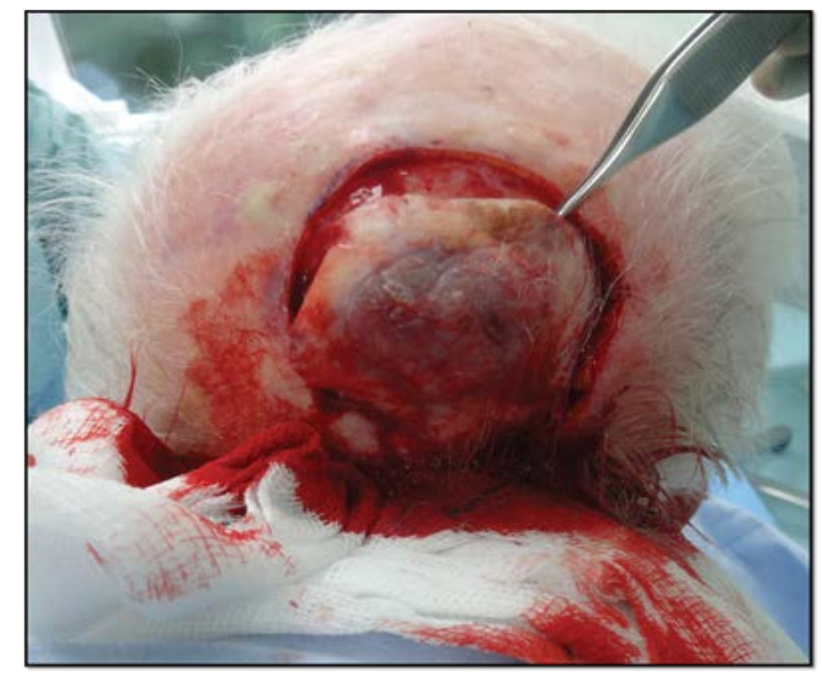

Figure 4: Tumour during excision.

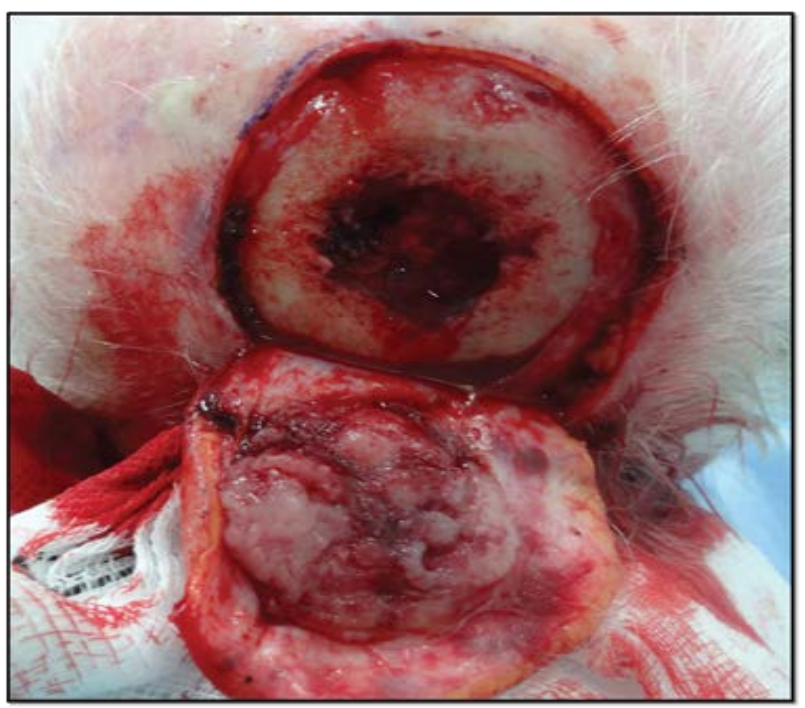

Figure 5: Extent of bony infiltration.

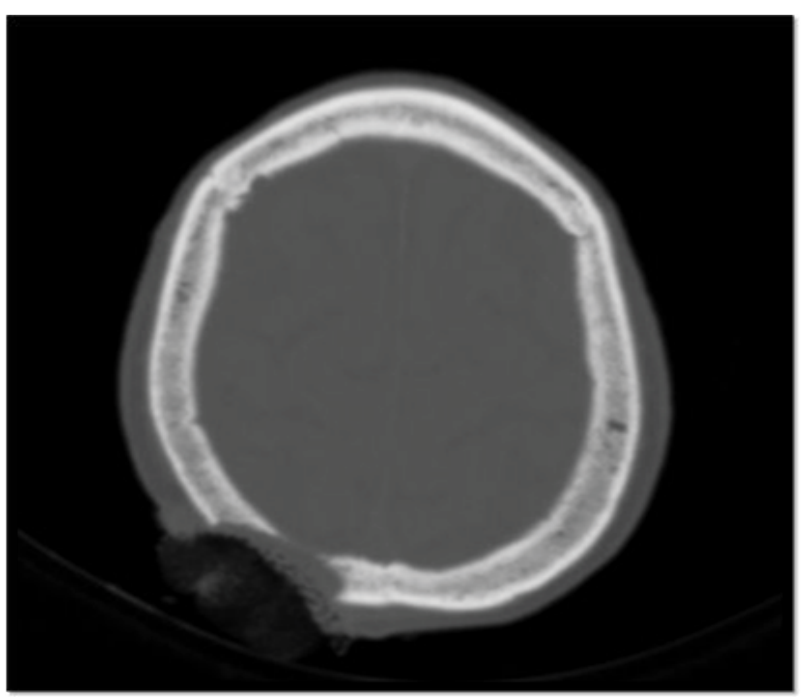

Figure 6: CT showing extent of skull invasion.

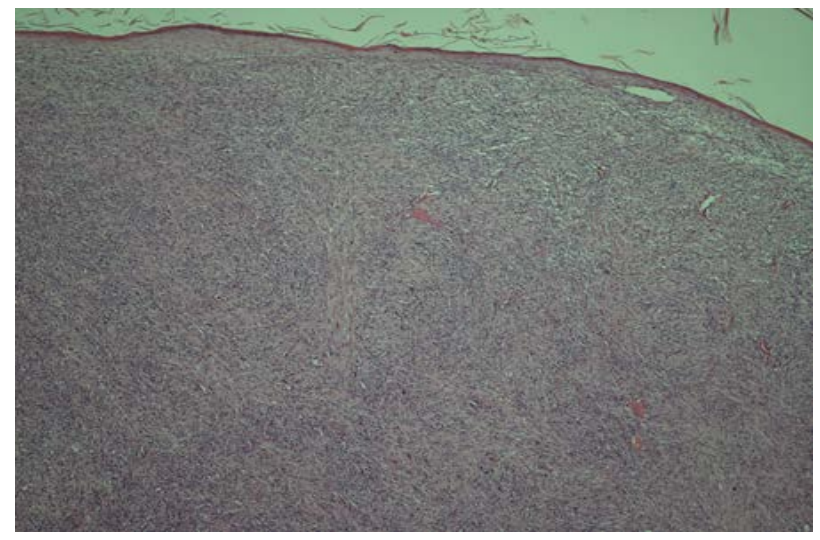

Figure 7: Histopathological image of the tumour (Light microscopy $x 4$; haematoxylin and eosin stain).

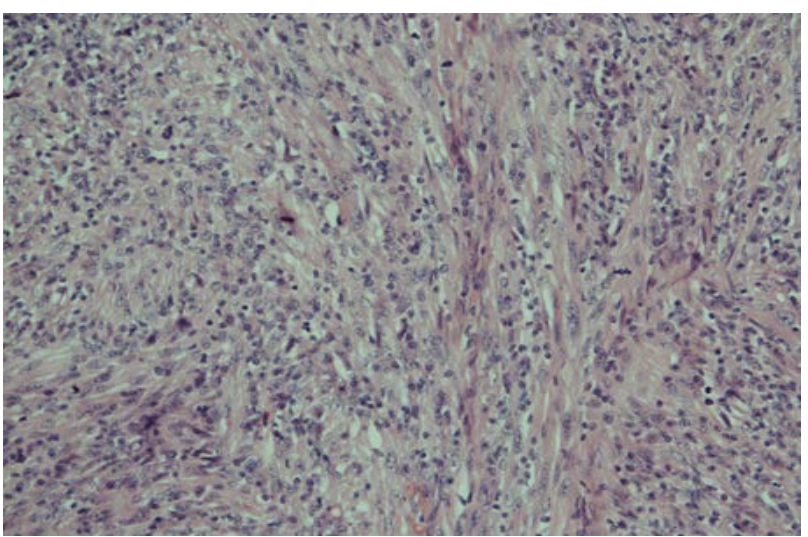

Figure 8: Histopathological image of the tumour, showing spindle cells arranged in storiform patterns and irregular fascicles with identifiable mitotic figures (Light microscopy x20; haematoxylin and eosin stain).

Histopathological examination revealed a large tumour of spindle cells arranged in storiform patterns and irregular fascicles, which focally show a moderate degree of pleomorphism (Figure 7 and Figure 8). There was also rather frequent mitosis identified. The whole tumour was admixed with frequent lymphocytes. It extended through the full thickness of the specimen and had invaded at least through the subcutaneous tissue to a thickness of $19 \mathrm{~mm}$. There was no evidence of vascular or perineural invasion. Immunohistochemistry showed positive staining for CD99 and CD10 and no staining for S100, Melan A, HMB45, MNF116, CK5, AE1/3, SMA, Desmin and Caldesmon. Histiocytes stained with CD68 were however rather frequent admixed with the tumour.

The specimen sent of calvarial bone contained more tumour and some fragments of bone admixed with it. The peripheral margins were clear by approximately $6 \mathrm{~mm}$, but the deep margin appeared to be widely involved.

Given the location, degree of invasion, morphology and immunohistochemical findings, the diagnosis was of Pleomorphic Dermal Sarcoma with bone involvement. 
Citation: Kapasi F, Cabral M, Ameerally P, et al. (2018) Case Report: Atypical Fibroxanthoma and Pleomorphic Dermal Sarcoma - Two Stages of the Same Disease?. Arch Surg Dermatol 1(1):13-17

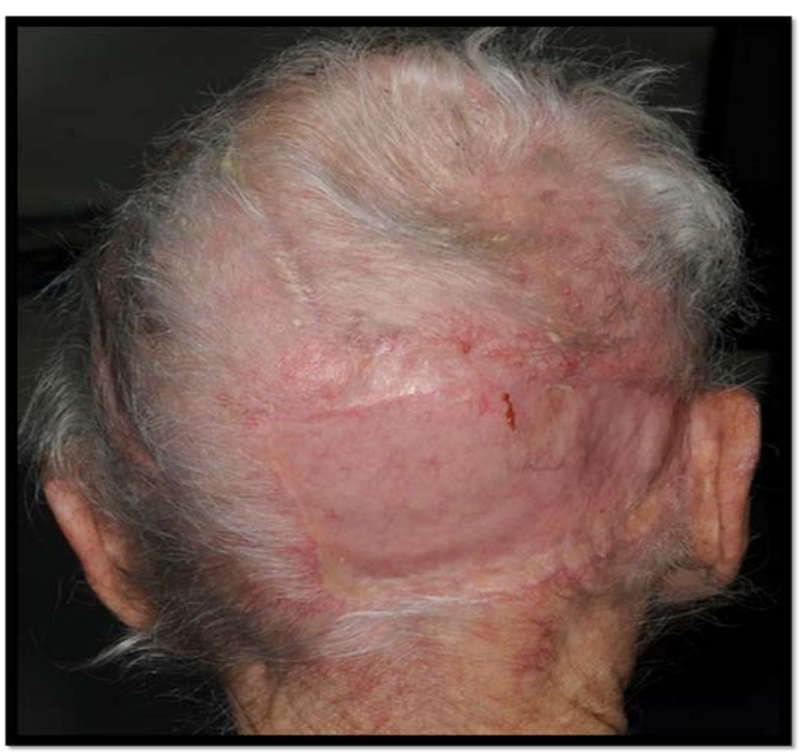

Figure 9: 6 months post-operatively.

The excision was incomplete. A referral was made to the joint care of Craniofacial Plastic Surgery and Neurosurgery and staging CT scans of the head, neck, thorax and abdomen and an MRI of the head were performed.

MRI demonstrated tumour with invasion through the scalp and full thickness of the skull with dural involvement. There were no further changes in CT in comparison to the previous scan. There were no distant bony metastases or cervical and thoracic lymphadenopathy.

In November 2016, the patient underwent excision of the scalp lesion, including affected cranium and dura. Dura was repaired with fascia lata graft and tissue patch, and the scalp defect was reconstructed with a transposition flap to cover the soft tissue defect and a split skin graft to the secondary defect (Figure 9).

There was no histological evidence of residual malignancy. There were areas of solar elastosis and hyperkeratosis on the skin. Beneath the ulcer there was fibrin, granulation tissue and heavy chronic and focally acute inflammation. The histological analysis of the bone specimen demonstrated replacement by granulation tissue containing heavy acute and chronic inflammatory infiltrate, haemosiderin deposits and foreign body type giant cells.

\section{Discussion}

The differential diagnoses of Atypical Fibroxanthoma include atypical fibrous histiocytoma, pleomorphic dermal sarcoma, dermatofibrosarcoma protuberans, squamous cell carcinoma, malignant melanoma, leiomyosarcoma, pleomorphic angiosarcoma and myofibrosarcoma [20].

This presentation of a rapidly growing papule in the scalp of an elderly patient with a history of multiple ac- tinic keratosis would be suggestive of BCC, SCC, Atypical Fibroxanthoma or Pleomorphic Dermal Sarcoma.

The histopathological examination of the initially excised lesion confirmed a diagnosis of Atypical Fibroxanthoma due to: The absence of extension beyond the dermis; presence of highly atypical pleomorphic and spindled cells showing frequent mitosis, which are the classic histological appearances; [17] and the absence of cytokeratin, desmin, S100, and CD34 expression which is a diagnostic criterion [17].

Wide local excision has been the standard treatment for Atypical Fibroxanthoma, with recommended margins of at least 1 to $2 \mathrm{~cm}$ to acquire the highest potential clearance [11,12]. However, Mohs Micrographic Surgery has been shown to provide superior clearance rates over wide local excision [21]. Other suggested therapies, such as cryotherapy and irradiation are discouraged [2].

Atypical Fibroxanthoma is regarded as a low-grade malignancy with a low rate of recurrence, likely around $10 \%$ with wide local excision [14]. Following a prolonged healing period, the initial lesion recurred and progressed rapidly. During excision, it was found to have extensive invasion with involvement of the underlying bone. Histopathological examination revealed a diagnosis of Pleomorphic Dermal Sarcoma with bone involvement.

Our case reports an Atypical Fibroxanthoma initially completely excised which recurred as a Pleomorphic Dermal Sarcoma with bone invasion. This raises the question that the low-grade behaviour and low recurrence rate of Atypical Fibroxanthoma may be underestimated and wider excision margins and closer monitoring with longer follow-up periods may be required.

This case also strongly reinforces the hypotheses that Atypical Fibroxanthoma and Pleomorphic Dermal Sarcoma represent two stages of the same disease, suggesting that the former may indeed be the precursor lesion of Pleomorphic Dermal Sarcoma.

\section{Fundling Sources}

None.

\section{IRB Approval Status}

Not required.

\section{Conflicts of Interest}

None declared.

\section{Reprint Requests}

Farhana Kapasi

\section{References}

1. Helwig EB (1963) Atypical fibroxanthoma: Proceedings of 
Citation: Kapasi F, Cabral M, Ameerally P, et al. (2018) Case Report: Atypical Fibroxanthoma and Pleomorphic Dermal Sarcoma - Two Stages of the Same Disease?. Arch Surg Dermatol 1(1):13-17

the 18th Annual Tumor Seminar San Antonio Society of Pathologists, 1961. Tex State J Med 59: 664-667.

2. Love W, Schmitt A, Bordeaux J (2011) Management of unusual cutaneous malignancies: Atypical fibroxanthoma, malignant fibrous histiocytoma, sebaceous carcinoma, extramammary Paget disease. Dermatol Clin 29: 201-216.

3. López L, Vélez R (2016) Atypical fibroxanthoma. Arch Pathol Lab Med 140: 376-379.

4. Inskip M, Magee J, Weedon D, et al. (2014) Atypical fibroxanthoma of the cheek-case report with dermatoscopy and dermatopathology. Dermatol Pract Concept 4: 77-80.

5. Nonaka D, Bishop P (2014) Sarcoma-like tumor of head and neck skin. Am J Surg Pathol 38: 956-965.

6. Singh M, Mann R, llankovan V, et al. (2012) Atypical fibroxanthoma-A retrospective immunohistochemical study of 42 cases. J Oral Maxillofac Surg 70: 2713-2718.

7. Hiscutt E, Adams J, Ryan J, et al. (2009) Atypical fibroxanthoma, lentigo maligna melanoma and squamous carcinoma arising in the site of a thermal burn treated with skin grafts. Br J Oral Maxillofac Surg 47: 157-158.

8. McCoppin HH, Christiansen D, Stasko T, et al. (2012) Clinical spectrum of atypical fibroxanthoma and undifferentiated pleomorphic sarcoma in solid organ transplant recipients: A collective experience. Dermatol Surg 38: 230-239.

9. Miller K, Goodlad J, Brenn T (2012) Pleomorphic dermal sarcoma: Adverse histologic features predict aggressive behavior and allow distinction from atypical fibroxanthoma. Am J Surg Pathol 36: 1317-1326.

10. Beer T, Drury P, Heenan P (2010) Atypical fibroxanthoma: A histological and immunohistochemical review of 171 cases. Am J Dermatopathol 32: 533-540.

11. González-García R, Nam-Cha S, Muñoz-Guerra M, et al. (2007) Atypical fibroxanthoma of the head and neck: report of 5 cases. J Oral Maxillofac Surg 65: 526-531.
12. Wollina U, Schönlebe J, Koch A, et al. (2010) Atypical fibroxanthoma: A series of 25 cases. J Eur Acad Dermatol Venereol 24: 943-946.

13. Wylie J, Hampton N, Telfer M, et al. (2010) Atypical fibroxanthoma: Case series of 16 patients. $\mathrm{Br} \mathrm{J}$ Oral Maxillofac Surg 48: 466-468.

14. Green B, Godden D, Brennan P (2015) Malignant cutaneous adnexal tumours of the head and neck: An update on management. British Journal of Oral and Maxillofacial Surgery 53: 485-490.

15. British Association of Dermatologists (2017) Atypical Fibroxanthoma: Patient Information Leaflet.

16. New D, Bahrami S, Malone J, et al. (2010) Atypical fibroxanthoma with regional lymph node metastasis: Report of a case and review of the literature. Arch Dermatol 146: 13991404.

17. Zschoche C, Hamsch C, Kutzner H, et al. (2014) Analysis of the lymphatic vessel architecture of atypical fibroxanthoma and pleomorphic dermal sarcoma. J Am Acad Dermatol 71: 842-845.

18. Helbig D, Ihle M, Pütz K, et al. (2016) Oncogene and therapeutic target analyses in atypical fibroxanthomas and pleomorphic dermal sarcomas. Oncotarget 7: 21763-21774.

19. Soleymani T, Hollmig S (2017) Conception and management of a poorly understood spectrum of dermatologic neoplasms: Atypical fibroxanthoma, pleomorphic dermal sarcoma, and undifferentiated pleomorphic sarcoma. Curr Treat Options Oncol 18: 50.

20. Hussein MR (2014) Atypical fibroxanthoma: New insights. Expert Rev Anticancer Ther 14: 1075-1088.

21. Davis JL, Randle HW, Zalla MJ, et al. (1997) A comparison of Mohs micrographic surgery and wide excision for the treatment of atypical fibroxanthoma. Dermatol Surg 23 . 105-110. 